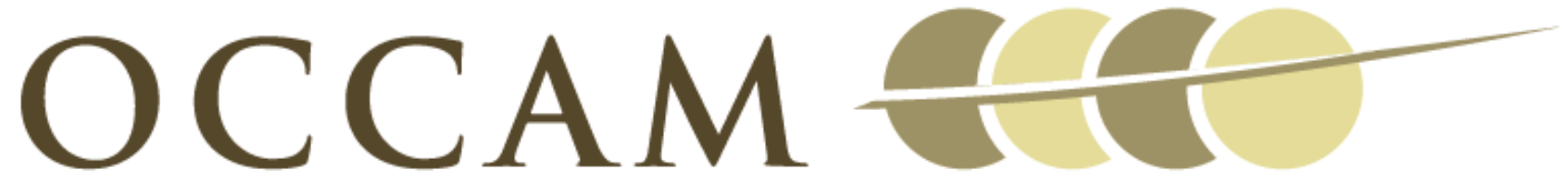

OXFORD CENTRE FOR COLLABORATIVE APPLIED MATHEMATICS

Report Number 10/61

Metastable states and quasicycles in a stochastic Wilson-Cowan model of neuronal population dynamics

by

Paul C. Bressloff

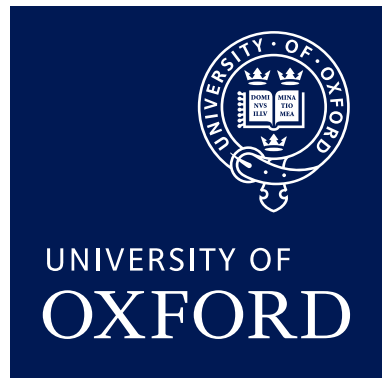

Oxford Centre for Collaborative Applied Mathematics Mathematical Institute 24 - 29 St Giles'

Oxford

OX1 3LB

England 



\title{
Metastable states and quasicycles in a stochastic Wilson-Cowan model of neuronal population dynamics
}

\author{
Paul C. Bressloff 1,2 \\ ${ }^{1}$ Mathematical Institute, University of Oxford, 24-29 St. Giles', Oxford OX1 3LB, UK \\ ${ }^{2}$ Department of Mathematics, University of Utah, 155 South, 1400 East, Salt lake City, UT 84112
}

(Dated: November 5, 2010)

\begin{abstract}
We analyze a stochastic model of neuronal population dynamics with intrinsic noise. In the thermodynamic limit $N \rightarrow \infty$, where $N$ determines the size of each population, the dynamics is described by deterministic Wilson-Cowan equations. On the other hand, for finite $N$ the dynamics is described by a master equation that determines the probability of spiking activity within each population. We first consider a single excitatory population that exhibits bistability in the deterministic limit. The steady-state probability distribution of the stochastic network has maxima at points corresponding to the stable fixed points of the deterministic network; the relative weighting of the two maxima depends on the system size. For large but finite $N$, we calculate the exponentially small rate of noise-induced transitions between the resulting metastable states using a WentzelKramers-Brillouin (WKB) approximation and matched asymptotic expansions. We then consider a two-population excitatory/inhibitory network that supports limit cycle oscillations. Using a diffusion approximation, we reduce the dynamics to a neural Langevin equation, and show how the intrinsic noise amplifies subthreshold oscillations (quasicycles).
\end{abstract}

PACS numbers: 87.19.lj, 87.19.lc

\section{INTRODUCTION}

In biochemical and gene networks within cells, there is an important distinction between intrinsic and extrinsic noise $[1,2]$. Extrinsic noise refers to external sources of randomness associated with environmental factors, and is often modeled as a continuous Markov process based on Langevin equations. On the other hand, intrinsic noise refers to random fluctuations arising from the discrete and probabilistic nature of chemical reactions at the molecular level, which are particularly significant when the number of reacting molecules $N$ is small. Under such circumstances, the traditional approach to modeling chemical reactions based on systems of ordinary differential equations that describe concentration changes via the law of mass action is inappropriate. Instead, a master equation formulation is necessary in order to describe the underlying jump Markov process. Since master equations are often difficult to analyze, diffusion approximations have been developed for mesoscopic systems (large but finite $N$ ) leading to a description based on the chemical Langevin equation [3, 4]. Although the diffusion approximation can capture the stochastic dynamics of mesoscopic reacting systems at finite times, it can break down in the limit $t \rightarrow \infty$. For example, suppose that the deterministic system based on the law of mass action (obtained in the thermodynamic limit $N \rightarrow \infty$ for finite $t$ ) has multiple stable fixed points. The diffusion approximation can then account for the effects of fluctuations well within the basin of attraction of a locally stable fixed point. However, there is now a small probability that there is a noise-induced transition to the basin of attraction of another fixed point. Since the probability of such a transition is usually of order $\mathrm{e}^{-\tau N}$ except close to the boundary of the basin of attraction, such a con- tribution cannot be analyzed accurately using standard Fokker-Planck methods [3]. These exponentially small transitions play a crucial role in allowing the network to approach the unique stationary state (if it exists) in the asymptotic limit $t \rightarrow \infty$. In other words, for multistable chemical systems, the limits $t \rightarrow \infty$ and $N \rightarrow \infty$ do not commute [5-7].

The distinction between intrinsic and extrinsic sources of noise can also be applied to neural systems, both at the single neuron and network levels $[8,9]$. It is well known that the spike trains of individual cortical neurons in vivo tend to be very noisy, having interspike interval (ISI) distributions that are close to Poisson [10]. The main source of intrinsic fluctuations is channel noise arising from the variability in the opening and closing of a finite number of ion channels. The resulting conductance-based model of a neuron can be formulated as a stochastic hybrid system, in which a continuous deterministic dynamics describing the time evolution of the membrane potential is coupled to a jump Markov process describing channel dynamics [11]. Extrinsic fluctuations at the single cell level are predominantly due to synaptic noise. That is, cortical neurons are bombarded by thousands of synaptic inputs, many of which are not correlated with a meaningful input and can thus be treated as background synaptic noise [9]. It is not straightforward to determine how noise at the single cell level translates into noise at the population or network level. One approach has been developed in terms of a Boltzmann-like kinetic theory of integrateand-fire networks $[12,13]$, which is itself a dimension reduction by moment closure of the population-density method $[14,15]$. The latter is a numerical scheme for tracking the probability density of a population of spiking neurons based on solutions of an underlying partial differential equation. In the case of simple neuron mod- 
els, this can be considerably more efficient than classical Monte Carlo simulations that follow the states of each neuron in the network. On the other hand, as the complexity of the individual neuron model increases, the gain in efficiency of the population density method decreases, and this has motivated the development of moment closure schemes. However, considerable care must be taken when carrying out the dimension reduction, since it can lead to an ill-posed problem over a wide range of physiological parameters [16]. That is, the truncated moment equations may not support a steady-state solution even though a steady-state probability density exists for the full system.

A number of studies of fully or sparsely connected integrate-and-fire networks have shown that under certain conditions, even though individual neurons exhibit Poisson-like statistics, the neurons fire asynchronously so that the total population activity evolves according to a mean-field rate equation with a characteristic activation or gain function [17-21]. Formally speaking, the asynchronous state only exists in the thermodynamic limit $N \rightarrow \infty$, where $N$ determines the size of the population. This then suggests a possible source of intrinsic noise at the network level arises from fluctuations about the asynchronous state due to finite size effects [22-25]. (Finite-size effects in IF networks have also been studied using linear response theory [26-28]). A closer analogy between intrinsic noise in biochemical and neural networks has recently been developed by the author [29], based on a rescaled version of the neural master equation introduced by Buice et. al. [30, 31], see also [32]. The underlying system consists of a network of coupled homogeneous populations. The state of each population is represented by the number of currently active neurons, and the state transitions are chosen such that deterministic Wilson-Cowan rate equations $[33,34]$ are recovered in the thermodynamic limit $N \rightarrow \infty$. Corrections to the mean-field equations involving higher-order moments can then be obtained by carrying out a VanKampen system size expansion along identical lines to chemical master equations [29]; such moment equations can also be obtained by carrying out a loop expansion of a corresponding path integral representation of the moment generating function [29-31].

In this paper we further develop the connection between chemical and neural master equations, by considering two important effects of intrinsic noise, namely, noise-induced transitions between metastable states, and noise-amplification of subthreshold oscillations or quasicycles. In both cases, we follow along analogous lines to previous studies of biochemical networks. In the case of noise-induced transitions, corrections to mean field theory based on the system-size or loop expansion break down. Instead, it is more appropriate to analyze the master equation using a WKB approximation and matched asymptotics $[5,35-39]$. On the other hand, quasicycles can be analyzed by considering the power spectrum of the associated Langevin equation obtained by carrying out a linear noise approximation of the master equation $[40,41]$. The structure of the paper is as follows. We introduce the birth-death master equation for a single bistable network in section II, and carry out the analysis of noise-induced transitions in section III. In section IV we present the master equation for a multiple population model, and analyze the noise-amplification of subthreshold oscillations in an excitatory/inhibitory network. We also briefly indicate how to extend the analysis of metastability to multiple populations. Finally, in section $\mathrm{V}$ we give some possible applications of our analysis.

\section{MASTER EQUATION FOR A BISTABLE ONE-POPULATION MODEL}

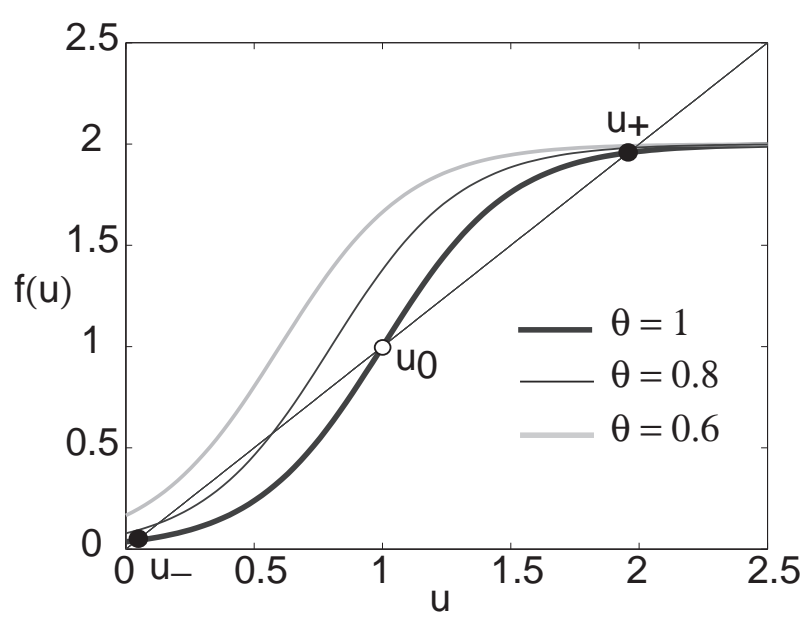

FIG. 1: Bistability in the deterministic network satisfying $\dot{u}=-u+f(u)$ with $f$ given by the sigmoid (2.2) for $\gamma=4$ and $\theta=1.0, f_{0}=2$. There exist two stable fixed points $u_{ \pm}$ separated by an unstable fixed point $u_{0}$. As the threshold $\theta$ is reduced the network switches to a monostable regime.

Consider a single homogeneous population of excitatory neurons satisfying a simple rate equation of the form

$$
\frac{d u}{d t}=-\alpha u+f(u)
$$

where $u$ is a measure of mean population activity, $\alpha$ is a rate constant and $f$ is a nonlinear firing rate function with gain $\gamma$ and threshold $\theta$. Note that for a single population model, the strength of recurrent connections within the population can be absorbed into the gain $\gamma$. For concreteness, $f$ is taken to be the sigmoid function

$$
f(u)=\frac{f_{0}}{1+\mathrm{e}^{-\gamma(u-\theta)}},
$$

with $f_{0}$ another rate constant. It is straightforward to show graphically that equation (2.1) exhibits bistability for a range of values of the gain and threshold. That 
is, there exist two stable fixed points $u_{ \pm}$separated by an unstable fixed point $u_{0}$, see Fig. 1 . We will fix the units of time by setting $\alpha=1$. (We interpret $\alpha^{-1}$ as a membrane time constant such that $\alpha^{-1}=10 \mathrm{msec}$ in physical units).

We now construct a stochastic version of the above rate model using a master equation formulation [29-32]. We assume that each neuron can be in either an active or quiescent state. Let $N(t)$ denote the number of active neurons at time $t$ and introduce the probability $P(n, t)=$ $\operatorname{Pr}\{N(t)=n\}$. The latter is taken to evolve according to a birth-death process of the form

$$
\frac{d P(n, t)}{d t}=T_{+}(n-1) P(n-1, t)+T_{-}(n+1) P(n+1, t)-\left(T_{+}(n)+T_{-}(n)\right) P(n, t)
$$

for integer $n \geq 0$, with boundary condition $P(-1, t) \equiv 0$ and initial condition $P(n, 0)=\delta_{n, \bar{n}}$. The birth and death rates are chosen to be of the form

$$
T_{+}(n)=N f(n / N), \quad T_{-}(n)=n,
$$

where $N$ is a system size parameter such that the deterministic rate equation (2.1) is recovered in the thermodynamic limit $N \rightarrow \infty$. That is, multiply both sides of the master equation (2.3) by $n$ and sum over $n$ to obtain

$$
\frac{d\langle n\rangle}{d t}=\left\langle T_{+}(n)\right\rangle-\left\langle T_{-}(n)\right\rangle,
$$

where the brackets $\langle\ldots\rangle$ denote a time-dependent ensemble averaging over realizations of the stochastic dynamics, that is, $\langle A(n)\rangle=\sum_{n} P(n, t) A(n)$ for any function of state $A(n)$. We now impose the mean-field approximation $\left\langle T_{ \pm}(n)\right\rangle \approx T_{ \pm}(\langle n\rangle)$, which is based on the assumption that statistical correlations can be neglected in the thermodynamic limit $N \rightarrow \infty$. Identifying mean population activity according to $u=\langle n\rangle / N$ then yields the rate equation (2.1). We leave the interpretation of the system size parameter $N$ open here. One possibility would be to identify $N$ with the number of neurons in the population such that $u(t)$ is the mean fraction of active neurons at time $t$ and $0 \leq u \leq 1$. However, $N$ could also be identified with the mean number of synaptic inputs into a neuron in a sparsely coupled network, for example. In this case the number of neurons in the network would be $\chi N$ for some integer $\chi$, where $\chi$ determines the degree of sparseness.

Note that the master equation (2.3) is a rescaled version of the one considered by Buice et. al. [30,31]. That is, they take the transition rate $T_{+}(n)=f(n)$ so that $u=\langle n\rangle$ in the mean-field equation (2.1), which corresponds to the mean number of active neurons. Such a choice would be appropriate if population activity were itself Poisson-like, rather than characterized in terms of finite-size fluctuations about an asynchronous state. The advantage of our choice of scaling from an analytical viewpoint is that one can treat $N^{-1}$ as a small parameter and use large deviation theory to study escape from metastable states [35, 42, 43]. Moreover, the finite time behavior within the basin of attraction of a metastable state can be analyzed using a system-size expansion or diffusion approximation [3].

In order to describe the non-trivial effects of fluctuations for large but finite $N$, suppose that the deterministic network is in the bistable regime so that the system tends towards either the high or low activity stable fixed point as $t \rightarrow \infty$, depending on the initial condition. In the same parameter regime, the stochastic network will also tend towards one of these fixed points on a fast timescale, but will randomly switch between the basins of attraction of the two fixed points on a slower timescale that depends exponentially on the system-size $N$; the fixed points are thus only metastable in the presence of intrinsic noise. The state transitions play a crucial role in determining the slow approach of the probability distribution $P(n, t)$ to its unique steady-state $P_{S}(n)$. The equation for the steady-state distribution $P_{S}(n)$ can be written as [44]

$$
0=J(n+1)-J(n),
$$

with $J(n)$ the probability current,

$$
J(n)=T_{-}(n) P_{S}(n)-T_{+}(n-1) P_{S}(n-1) .
$$

Since $T_{-}(0)=0$ and $P_{S}(-1)=0$, it follows that $J(0)=0$ and $J(n)=0$ for all $n \geq 0$. Hence,

$$
P_{S}(n)=\frac{T_{+}(n-1)}{T_{-}(n)} P_{S}(n-1)=P_{S}(0) \prod_{m=1}^{n} \frac{T_{+}(m-1)}{T_{-}(m)}
$$

with $P_{S}(0)=1-\sum_{m \geq 1} P_{S}(m)$. One finds that the stable and unstable fixed points of the deterministic rate equation are represented as minima and maxima, respectively, of the steady-state distribution $P_{S}(n)$, see Fig. 2 . The relative heights of the maxima depend on the system size $N$ as illustrated in Fig. 3 .

Note that approximating the neural master equation (2.3) by a neural Langevin equation would lead to exponentially large errors in the values of the peaks of the steady-state distribution, and consequently exponentially large errors in the calculation of the escape rates between metastable states (see section 3). The Langevin equation can be derived by carrying out a KramersMoyal expansion of the master equation [3, 4]. First, 

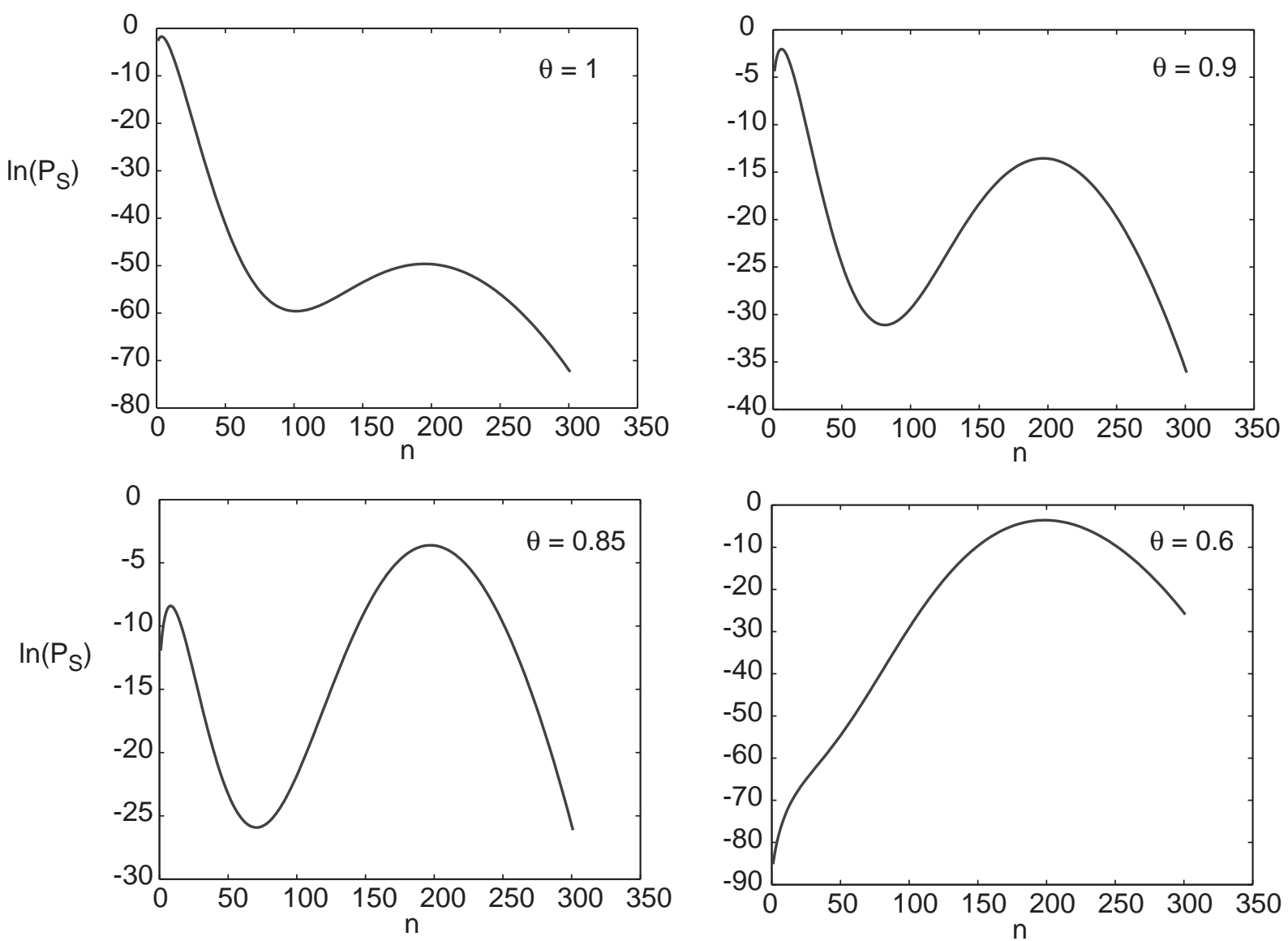

FIG. 2: Plots of the steady state distribution $P_{S}(n)$ for various thresholds $\theta$ with $\gamma=4.0, f_{0}=2$. System size is $N=100$.
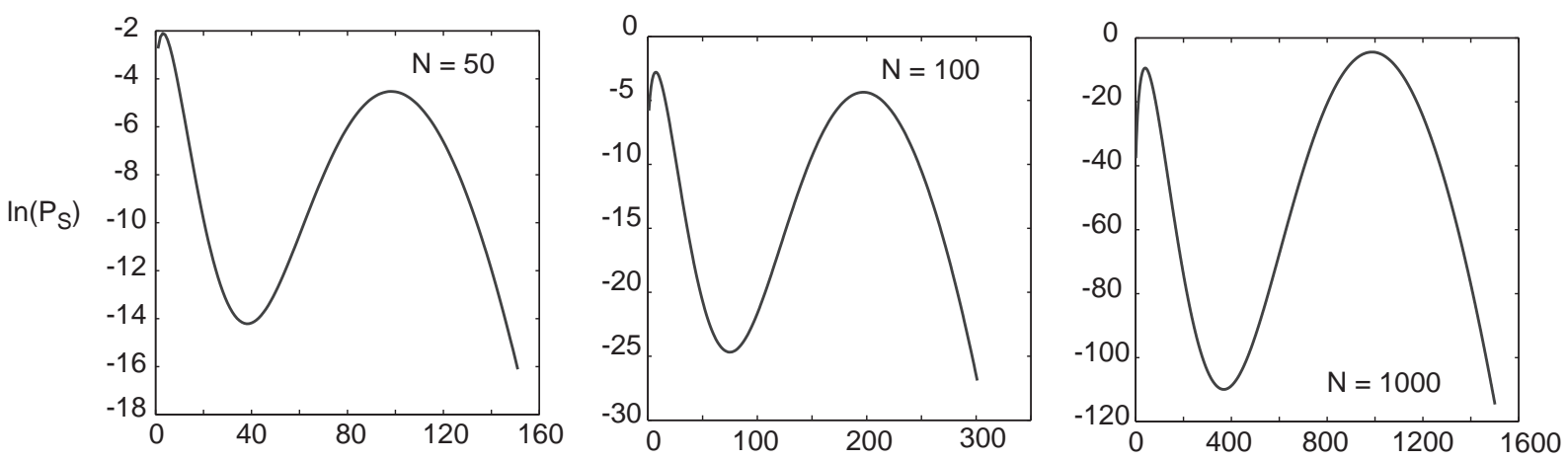

FIG. 3: Plots of steady state distribution $P_{S}(n)$ for various system sizes $N$. Other parameters are $\gamma=4, \theta=0.87, f_{0}=2$

introduce the rescaled variable $x=n / N$ and correspond- Equation (2.3) can then be rewritten in the form ing transition rates $N \Omega_{ \pm}(x)=T_{ \pm}(N x)$, that is,

$$
\Omega_{+}(x)=f(x), \quad \Omega_{-}(x)=x .
$$




$$
\frac{d P(x, t)}{d t}=N\left[\Omega_{+}(x-1 / N) P(x-1 / N, t)+\Omega_{-}(x+1 / N) P(x+1 / N, t)-\left(\Omega_{+}(x)+\Omega_{-}(x)\right) P(x, t)\right] .
$$

Treating $x$ as a continuous variable and Taylor expanding terms on the right-hand side to second order in $N^{-1}$ leads to the Fokker-Planck (FP) equation

$$
\begin{aligned}
\frac{\partial P(x, t)}{\partial t}= & -\frac{\partial}{\partial x}[A(x) P(x, t)] \\
& +\frac{\epsilon^{2}}{2} \sum_{k=1}^{M} \frac{\partial^{2}}{\partial x^{2}}[B(x) P(x, t)]
\end{aligned}
$$

with $\epsilon=N^{-1 / 2}$ and

$$
A(x)=\Omega_{+}(x)-\Omega_{-}(x), \quad B(x)=\Omega_{+}(x)+\Omega_{-}(x) .
$$

The solution to the FP equation (2.11) determines the probability density function for a corresponding Ito stochastic process $X(t)$, which evolves according to a neural Langevin equation of the form

$$
d X=A(X) d t+\epsilon b(X) d W(t)
$$

with $b(x)^{2}=B(x)$. Here $W(t)$ denotes an independent Wiener process such that

$$
\langle W(t)\rangle=0, \quad\langle W(t) W(s)\rangle=\min (t, s) .
$$

The FP equation (2.11) has the steady-state solution

$$
P_{S}(x)=\mathcal{A} \exp \left(2 N \int^{x} \frac{\Omega_{+}\left(x^{\prime}\right)-\Omega_{-}\left(x^{\prime}\right)}{\Omega_{+}\left(x^{\prime}\right)+\Omega_{-}\left(x^{\prime}\right)} d x^{\prime}\right),
$$

where $\mathcal{A}$ is a normalization factor. On the other hand, setting $n=N x$ in equation (2.8), one finds that for large $N$ (see also section 3 ),

$$
P_{S}(x)=\mathcal{A}^{\prime} \exp \left(N \int^{x} \ln \frac{\Omega_{+}\left(x^{\prime}\right)}{\Omega_{-}\left(x^{\prime}\right)} d x^{\prime}\right)
$$

with normalization factor $\mathcal{A}^{\prime}$. Writing $P_{S}(x) \sim \mathrm{e}^{-N \Phi(x)}$, we see that the stochastic potential $\Phi(x)$ associated with the FP equation differs from that of the underlying master equation, and this leads to exponentially large errors when calculating escape rates for example. Thus great care must be taken when approximating a neural (or chemical) master equation by a corresponding FP or Langevin equation [5-7].

\section{ANALYSIS OF STOCHASTIC DYNAMICS IN THE BISTABLE REGIME}

In order to explore the approach to steady-state, let us rewrite the master equation (2.3) as the linear system

$$
\frac{d \mathbf{p}}{d t}=\mathbf{Q p}
$$

where $\mathbf{p}=(P(0, t), P(1, t), \ldots)^{T}$ and $\mathbf{Q}$ is the matrix

$$
\mathbf{Q}=\left(\begin{array}{cccc}
-T_{+}(0) & T_{-}(1) & 0 & \cdots \\
T_{+}(0) & -T_{+}(1)-T_{-}(1) & T_{-}(2) & \cdots \\
0 & T_{+}(1) & -T_{+}(2)-T_{-}(2) & \cdots \\
\vdots & \vdots & \vdots & \vdots
\end{array}\right) .
$$

Suppose that we order the eigenvalues of $\mathbf{Q}$ according to $\lambda_{0} \geq \lambda_{1} \geq \lambda_{2} \geq \ldots$. The Perron-Frobenius theorem implies that all the eigenvalues of $\mathbf{Q}$ are real with the largest being a simple eigenvalue, $\lambda_{0}=0$, so that $\lambda_{i}<0$ for $i \geq 1$. Suppose that the system is operating in a bistable regime so that there exist two stable fixed points $x_{ \pm}$and one unstable fixed point $x_{0}$ of the corresponding deterministic rate equation (2.1). In the bistable regime, one finds that the second largest eigenvalue $\lambda_{1}$ differs from the other negative eigenvalues in the sense that it decays exponentially with the system size $N,\left|\lambda_{1}\right| \sim \mathrm{e}^{-\tau_{1} N}$ for $\tau_{1}=\mathcal{O}(1)$, whereas $\lambda_{i}, i>1$ are only weakly dependent on $N$. The existence of an exponentially small eigenvalue reflects the corresponding exponential dependence on $N$ of the escape rates between the two stable fixed points of the deterministic system. Indeed, $\lambda_{1}=-\left(r_{+}+r_{-}\right)$[5], where $r_{-}$is the escape rate from $x_{-}$to $x_{+}$and $r_{+}$is the escape rate from $x_{+}$to $x_{-}$. Expanding the solution to the master equation (2.3) in terms of the eigenvalues $\lambda_{i}$ and corresponding eigenvectors $v_{i}(n)$,

$$
P(n, t)=c_{0} v_{0}(n)+c_{1} v_{1}(n) \mathrm{e}^{\lambda_{1} t}+\cdots .
$$

Since $\lambda_{i}<0$ for all $i \geq 1$, it follows that $\lim _{t \rightarrow \infty} P(n, t)=$ $c_{0} v_{0}(n)$ and we can identify $v_{0}(n)$ with the steady-state distribution $P_{S}(n)$. The higher order eigenmodes $v_{i}(n)$ for $i>1$ will decay relatively rapidly so that the approach to the steady-state will be dominated by the first eigenmode $v_{1}(n)$. In this section we show how the escape rates $r_{ \pm}$and hence the eigenvalue $\lambda_{1}$ can be calculated by exploiting the fact that $r_{ \pm}$are exponentially small functions of the system size $N$. Following previous studies of chemical master equations [5, 35-39], we construct a quasistationary solution of the master equation using a WKB approximation. This leads to a corresponding Hamiltonian dynamical system that describes the most probable path of escape from a metastable state. The escape rates are then obtained by asymptotically matching the quasistationary solution with an appropriate inner solution in a neighborhood of the saddle point $x=x_{0}$. This is necessary in order to satisfy appropriate boundary conditions at $x_{0}$. Finally, the long-term behavior of the bistable network can be represented in terms of a two-state Markov process describing transitions between 
the two metastable states $n_{ \pm}=N x_{ \pm}[7]$.

\section{WKB approximation}

Let $\Pi(x)$ with $x=n / N$ denote a quasistationary solution associated with finding the network in the basin of attraction of one of the stable fixed points $x^{*}=x_{ \pm}$:

$$
\begin{aligned}
0= & \Omega_{+}(x-1 / N) \Pi(x-1 / N) \\
& +\Omega_{-}(x+1 / N) \Pi(x+1 / N)-\left(\Omega_{+}(x)+\Omega_{-}(x)\right) \Pi(x) .
\end{aligned}
$$

Note that here $x$ is treated as a continuous variable $x \in$ $[0, \infty)$. We seek a WKB solution of the form

$$
\Pi(x) \sim K(x) \mathrm{e}^{-N S(x)},
$$

with $S\left(x^{*}\right)=0, K\left(x^{*}\right)=1$. Substituting equation (3.5) into equation (3.4), Taylor expanding with respect to $N^{-1}$, and collecting the $\mathcal{O}(1)$ terms gives

$$
\sum_{r= \pm 1} \Omega_{r}(x)\left[\mathrm{e}^{r S^{\prime}(x)}-1\right]=0
$$

where $S^{\prime}=d S / d x$. Mathematically speaking, equation (3.6) takes the form of a stationary Hamilton-Jacobi equation $H\left(x, S^{\prime}(x)\right)=0$ for $S$, with Hamiltonian

$$
\begin{aligned}
H(x, p) & =\sum_{r= \pm 1} \Omega_{r}(x)\left[\mathrm{e}^{r p}-1\right] \\
& =x\left[\mathrm{e}^{-p}-1\right]+f(x)\left[\mathrm{e}^{p}-1\right] .
\end{aligned}
$$

This suggests a corresponding classical mechanical interpretation, in which $H$ determines the motion of a particle with position $x$ and conjugate momentum $p$. A trajectory of the particle is given by the solution of Hamilton's equations

$$
\begin{aligned}
\dot{x} & =\frac{\partial H}{\partial p}=\sum_{r= \pm 1} r \Omega_{r}(x) \mathrm{e}^{r p} \\
& =-x \mathrm{e}^{-p}+f(x) \mathrm{e}^{p} \\
\dot{p} & =-\frac{\partial H}{\partial x}=\sum_{r= \pm 1} \frac{\partial \Omega_{r}}{\partial x}(x)\left[\mathrm{e}^{r p}-1\right] \\
& =\left[\mathrm{e}^{-p}-1\right]+f^{\prime}(x)\left[\mathrm{e}^{p}-1\right]
\end{aligned}
$$

Here the time $t$ should be viewed as a parameterization of paths rather than as a real time variable. Introducing the Lagrangian

$$
L(x, \dot{x})=p \cdot \dot{x}-H(x, p),
$$

it follows that $S(x)$ with $S\left(x^{*}\right)=0$ corresponds to the classical action evaluated along the least-action trajectory from $x^{*}$ to $x$ :

$$
S(x)=\inf _{x\left(t_{0}\right)=x^{*}, x(T)=x} \int_{0}^{T} L(x, \dot{x}) d t .
$$

In terms of the underlying stochastic process $X(t)$, the least-action path can be interpreted as the most probable fluctuational path from $x^{*}$ to $x$ (in the large $-N$ limit) $[42,43]$. Since $p=S^{\prime}$ everywhere along this path, we have

$$
S(x)=\int_{x^{*}}^{x} p\left(x^{\prime}\right) d x^{\prime}
$$

with the integral taken along the trajectory. It follows that the leading order term in the WKB approximation is determined by finding zero-energy solutions $p=p(x)$ such that $H(x, p(x))=0$. Given these zero energy solutions, the multiplicative factor $K(x)$ can be determined by solving the equation

$$
H_{p} \frac{K^{\prime}}{K}=-\frac{1}{2} p^{\prime} H_{p p}-H_{p x}
$$

with $H_{p}=\partial H(x, p(x)) / \partial p$ etc. Equation (3.13) is obtained by collecting the $\mathcal{O}(1 / N)$ terms in the WKB solution of equation (3.4)

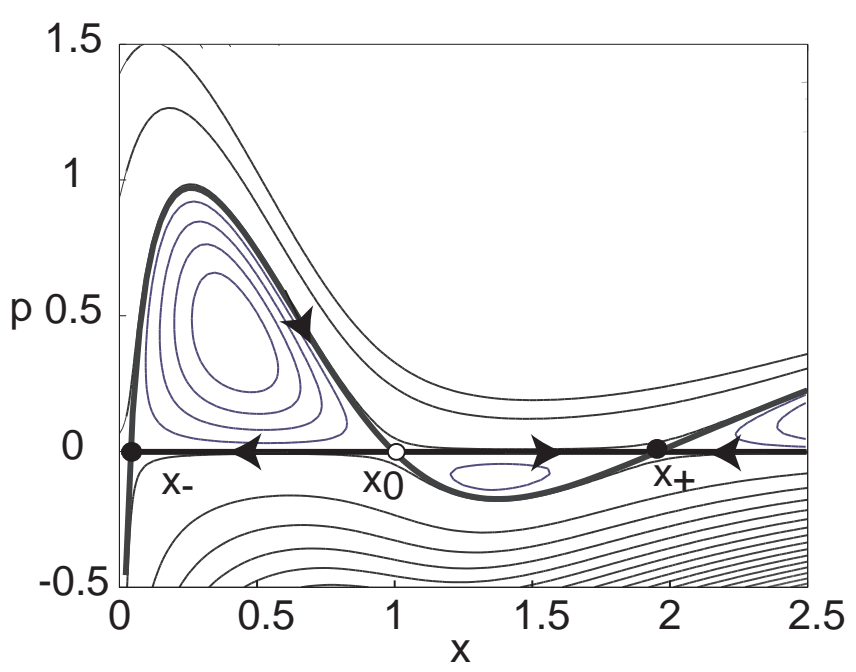

FIG. 4: Phase portrait of Hamiltonian equations of motion for $\Omega_{+}(x)=f_{0} /\left(1+\mathrm{e}^{-\gamma(x-\theta)}\right)$ and $\Omega_{-}(x)=x$ with $\gamma=4, \theta=1.0$ and $f_{0}=2$. The zero energy solutions are shown as thicker curves.

Since equation (3.6) is a quadratic in $\mathrm{e}^{p}$, there are two classes of zero-energy solution given by (see Fig. 4)

$$
p=0, \quad p=p_{*}(x) \equiv \ln \frac{\Omega_{-}(x)}{\Omega_{+}(x)} .
$$

The classical equation of motion along the so-called relaxation trajectory $p=0$ is precisely the deterministic rate equation (2.1), which in the bistable regime has three fixed points $x_{ \pm}, x_{0}$. Motion along a neighborhood of a relaxation trajectory towards one of the two fixed points $x_{ \pm}$characterizes the fast behavior of the system that is consistent with a Gaussian process in a neighborhood of the given fixed point. However, on exponentially long time-scales we expect transitions between 
the two metastable states centered about $x_{ \pm}$, and the most likely (optimal) path of escape will be along the so-called activation trajectory $p=p_{*}(x)$ from $x_{ \pm}$to the saddle point $x_{0}$. On crossing $x_{0}$ we expect the system to rapidly move along the relaxation trajectory to the other fixed point $x_{\mp}$. In Fig. 4 we illustrate the Hamiltonian phase space for a bistable neural network showing the constant energy solutions of the Hamiltonian given by equation (3.7); the zero energy activation and relaxation trajectories through the fixed points of the deterministic system are highlighted as thicker curves. After evaluating the factor $K(x)$ by solving equation (3.13), it follows that along an activation trajectory [39]

$$
\Pi(x)=\frac{A}{\sqrt{\Omega_{+}(x) \Omega_{-}(x)}} \mathrm{e}^{-N S(x)} .
$$

with

$$
S(x)=\int^{x} \ln \frac{\Omega_{-}(y)}{\Omega_{+}(y)} d y,
$$

whereas, along a relaxation trajectory $S=0$ and

$$
\Pi(x)=\frac{B}{\Omega_{+}(x)-\Omega_{-}(x)}
$$

for some constant $B$.

\section{Calculation of escape rates}

Given the WKB approximation, the rate of escape $r_{-}$ from the metastable state centered about $x=x_{-}$can be calculated by matching the quasistationary solutions with an appropriate inner solution in a neighborhood of the saddle point $x=x_{0}[5,35-39]$. This is necessary since the quasistationary solutions (3.15) and (3.17) do not satsify appropriate boundary conditions at the saddle point separating the two metastable states. We will briefly describe the method of Escudero and Kamanev [39], and then apply their result to the particular example of a bistable neural network. Escudero and Kamanev assume that there is a constant flux $J$ through the saddle and then match the activation solution on one side of the saddle $\left(x<x_{0}\right)$ with the relaxation solution on the other side of the saddle $\left(x>x_{0}\right)$ using a diffusion approximation of the full master equation (2.3) in the vicinity of $x_{0}$. The latter yields the Fokker-Planck equation (2.11), which can be rewritten in the form of a continuity relation

$$
\frac{\partial}{\partial t} P(x, t)=-\frac{\partial}{\partial x} J(x, t)
$$

with

$$
\begin{aligned}
J(x, t)= & \left(\Omega_{+}(x)-\Omega_{-}(x)\right) P(x, t) \\
& -\frac{1}{2 N} \frac{\partial}{\partial x}\left[\left(\Omega_{+}(x)+\Omega_{-}(x)\right) P(x, t)\right] .
\end{aligned}
$$

Substituting the quasistationary solution $\Pi(x, t)=$ $\Pi(x) \mathrm{e}^{-r_{-} t}$ into equation (3.18) and using the fact that $r_{-}$is exponentially small, gives

$$
\begin{aligned}
J= & \left(\Omega_{+}(x)-\Omega_{-}(x)\right) \Pi(x) \\
& -\frac{1}{2 N} \frac{\partial}{\partial x}\left[\left(\Omega_{+}(x)+\Omega_{-}(x)\right) \Pi(x)\right],
\end{aligned}
$$

where $J$ is the constant flux through the saddle. In a neighborhood of the saddle this equation can be Taylor expanded to leading order in $x-x_{0}$ and integrated to obtain the solution

$$
\Pi(x)=\frac{J N}{\Omega_{+}\left(x_{0}\right)} \mathrm{e}^{\left(x-x_{0}\right)^{2} / \sigma^{2}} \int_{x}^{\infty} \mathrm{e}^{-\left(y-x_{0}\right)^{2} / \sigma^{2}} d y,
$$

where

$$
\sigma=\sqrt{\frac{2 \Omega_{+}\left(x_{0}\right)}{N\left[\Omega_{+}^{\prime}\left(x_{0}\right)-\Omega_{-}^{\prime}\left(x_{0}\right)\right]}}
$$

determines the size of the boundary layer around the saddle.

In order to match the activation and relaxation solutions, the following asymptotic behavior of the inner solution (3.21) is used:

$$
\Pi(x)=\left\{\begin{array}{cc}
\frac{N J \sigma^{2}}{\left(x-x_{0}\right) \Omega_{+}\left(x_{0}\right)}, & x-x_{0} \gg \sigma \\
\frac{N J \sigma \sqrt{\pi}}{\Omega_{+}\left(x_{0}\right)} \mathrm{e}^{\left(x-x_{0}\right)^{2} / \sigma^{2}}, & x_{0}-x \gg \sigma
\end{array} .\right.
$$

The solution to the right of the saddle matches the relaxation solution (3.17) since $\Omega_{+}(x)-\Omega_{-}(x) \approx(x-$ $\left.x_{0}\right)\left[\Omega_{+}^{\prime}\left(x_{0}\right)-\Omega_{-}^{\prime}\left(x_{0}\right)\right]$ for $x \approx x_{0}$ such that $B=J$. In order to match the solution on the left-hand side of the saddle with the activation solution (3.15), Taylor expand $S(x)$ about $x_{0}$ using $S^{\prime}\left(x_{0}\right)=0$ and $S^{\prime \prime}\left(x_{0}\right)=-2 / N \sigma^{2}$. It follows that

$$
J=\frac{A \Omega_{+}\left(x_{0}\right)}{\sqrt{\Omega_{+}\left(x_{0}\right) \Omega_{-}\left(x_{0}\right)}} \sqrt{\frac{\left|S^{\prime \prime}\left(x_{0}\right)\right|}{2 \pi N}} \mathrm{e}^{-N S\left(x_{0}\right)} .
$$

The final step in the analysis is to link the flux $J$ with the escape rate $r_{-}$. This is achieved by integrating the continuity equation (3.18) over the interval $x \in\left[0, x_{0}\right]$ and using a reflecting boundary condition at $x=0$ :

$$
\frac{1}{r_{-}}=\frac{1}{J} \int_{0}^{x_{0}} \Pi(y) d y .
$$

Since the activation solution is strongly peaked around the fixed pont $x_{-}$, a Gaussian approximation of $\Pi(x)$ around $x_{-}$can be used to give the result [39]

$$
r_{-}=\frac{\Omega_{+}\left(x_{-}\right)}{2 \pi} \sqrt{\left|S^{\prime \prime}\left(x_{0}\right)\right| S^{\prime \prime}\left(x_{-}\right)} \mathrm{e}^{-N\left[S\left(x_{0}\right)-S\left(x_{-}\right)\right]} .
$$




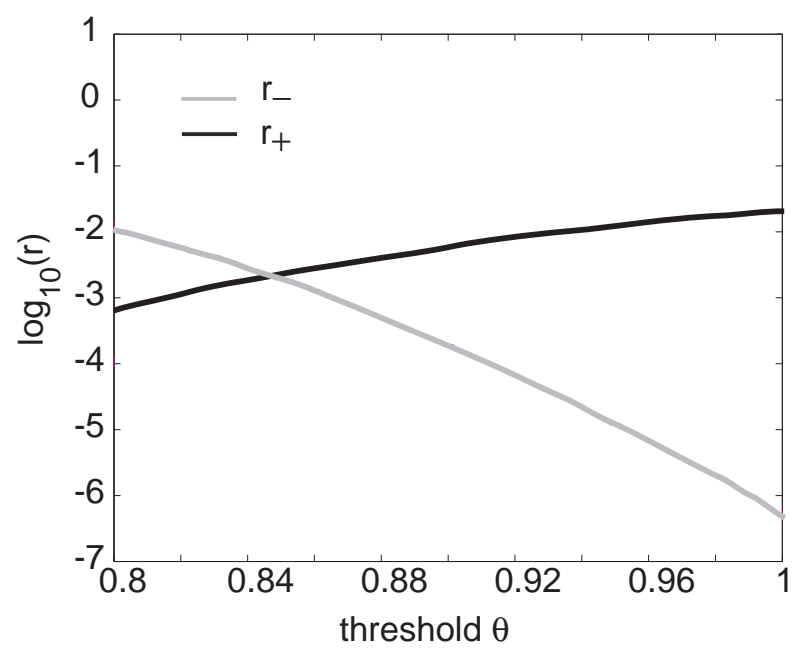

FIG. 5: Plot of calculated escape rates $r_{ \pm}$for a bistable neural network as a function of the threshold $\theta$. Other parameters are $\gamma=4.0, f_{0}=2$ and $N=20$. The escape rates are approximately equal for $\theta \approx 0.85$.

Similarly, the escape rate from the metastable state $x_{+}$ is

$$
r_{+}=\frac{\Omega_{+}\left(x_{+}\right)}{2 \pi} \sqrt{\left|S^{\prime \prime}\left(x_{0}\right)\right| S^{\prime \prime}\left(x_{+}\right)} \mathrm{e}^{-N\left[S\left(x_{0}\right)-S\left(x_{+}\right)\right]} .
$$

We now apply the above results to the particular case of a bistable neural network evolving according to a master equation with transition rates (2.9). In particular, the variation of the calculated escape rates $r_{ \pm}$with respect to the threshold $\theta$ is plotted in Fig. 5. As expected, $r_{+}\left(r_{-}\right)$increases (decreases) as the threshold increases, reflecting the fact that the more active metastable state dominates at lower thresholds. It can also be seen that there is a narrow range of thresholds over which $r_{ \pm}$are comparable, otherwise one metastable state dominates. The bistable nature of the stochastic neural network is illustrated in Figs. 6 and 7 for different choices of threshold. It can be seen that the dynamics is characterized by sharp transitions between the two metastable states $x_{ \pm}$ combined with localized (Gaussian-like) fluctuations in a neighborhood of each state. Consistent with 5, lowering the threshold increases the relative time the network spends in the active state. (Note that the bistable nature of the system vanishes in the rescaled version of the master equation considered by Buice et. al. [30, 31], since it operates in a Poisson-like regime).

\section{Bistable network as a two-state Markov process}

Having calculated the escape rates $r_{ \pm}$, the long-term behavior of the stochastic bistable network can be approximated by a two-state Markov process that only keeps track of which metastable state the system is close

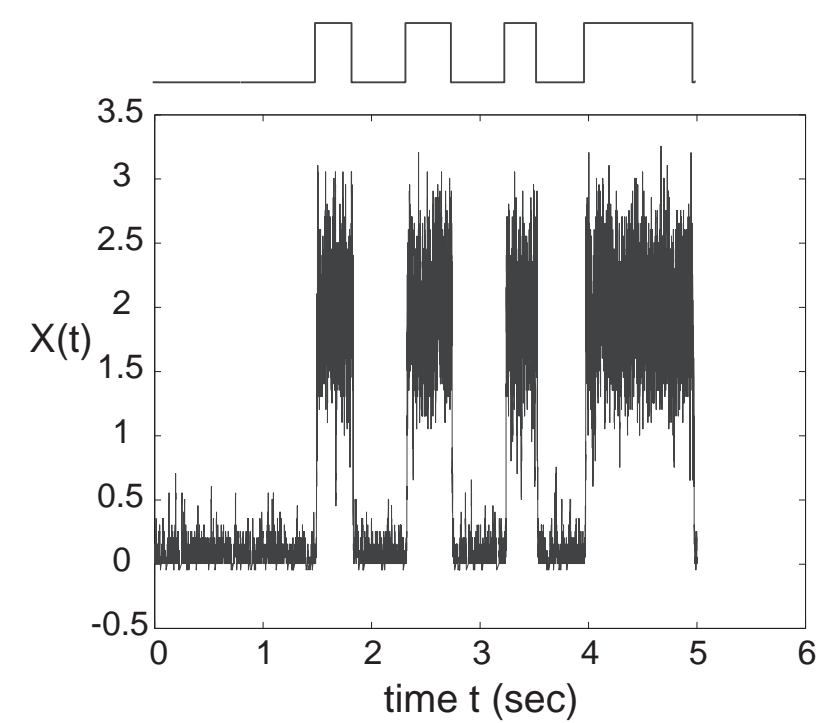

FIG. 6: Time series showing a single realization of the stochastic process $X(t)=N(t) / N$ where $N(t)$ is the birth death process corresponding to the neural master equation (2.3). Parameters are $\theta=0.86, \gamma=4.0, f_{0}=2$ and $N=20$. The bistable nature of the process is clearly seen.

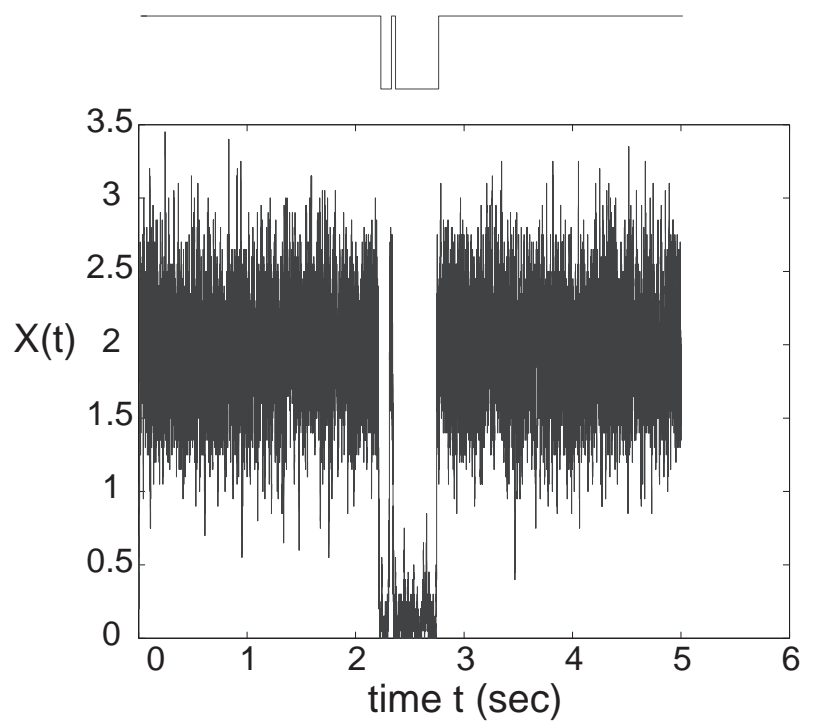

FIG. 7: Same as Fig. 6 except that $\theta=0.83$.

to $[7]$ :

$$
\frac{d}{d t}\left(\begin{array}{l}
P_{-} \\
P_{+}
\end{array}\right)=\widehat{\mathbf{Q}}\left(\begin{array}{l}
P_{-} \\
P_{+}
\end{array}\right), \quad \widehat{\mathbf{Q}}=\left(\begin{array}{cc}
-r_{-} & r_{+} \\
r_{-} & -r_{+}
\end{array}\right)
$$

where $P_{ \pm}$are the probabilities of being in a neighborhood of $x_{ \pm}$. The matrix $\widehat{\mathbf{Q}}$ has eigenvectors $\widehat{\lambda}_{0}=0$ and $\widehat{\lambda}_{1}=$ $-\left(r_{+}+r_{-}\right)$and corresponding eigenvectors

$$
\widehat{\mathbf{v}}_{0}=\left(\begin{array}{c}
\frac{r_{+}}{r_{+}+r_{-}} \\
\frac{r_{+}+r_{-}}{r_{+}}
\end{array}\right), \quad \widehat{\mathbf{v}}_{1}=\left(\begin{array}{c}
\frac{1}{2} \\
-\frac{1}{2}
\end{array}\right) .
$$


The eigenvalues of $\widehat{\mathbf{Q}}$ are identical to the first two eigenvalues of the full matrix $\mathbf{Q}$ of the master equation (3.1). Moreover, the two-component eigenvectors $\widehat{\mathbf{v}}_{j}$ are reduced versions of the eigenvectors $v_{j}(n), n \geq 0$ of $\mathbf{Q}$. That is,

$$
\widehat{v}_{j, 1} \approx \sum_{n=0}^{n_{0}} v_{j}(n), \quad \widehat{v}_{j, 2} \approx \sum_{n=n_{0}}^{\infty} v_{j}(n), \quad j=0,1
$$

In particular, $\widehat{\mathbf{v}}_{0}$ determines the steady-state probability of being in the neighborhood of either metastable state. Note that the two-state model generates an exponential density for the residence times within a given metastable state. This captures the behavior of the full master equation at large residence times but fails to capture the short-term dynamics associated with relaxation trajectories within a neighborhood of the metastable state.

\section{MULTIPLE POPULATION MODEL}

Now suppose that there exist $M$ homogeneous neuronal populations labeled $i=1, \ldots, M$, each of size $N$. Assume that all neurons of a given population are equivalent in the sense that the effective pairwise synaptic interaction between a neuron of population $i$ and a neuron of population $j$ only depends on $i$ and $j$. Suppose that there are $N_{k}(t)$ active neurons in the $k$ th population. The state or configuration of the network is now specified by the vector $\mathbf{N}(t)=\left(N_{1}(t), N_{2}(t), \ldots, N_{M}(t)\right)$, where each $N_{i}(t)$ is treated as a stochastic variable that evolves according to a one-step jump Markov process. Let $P(\mathbf{n}, t)=\operatorname{Prob}[\mathbf{N}(t)=\mathbf{n}]$ denote the probability that the network of interacting populations has configuration $\mathbf{n}=\left(n_{1}, n_{2}, \ldots, n_{M}\right)$ at time $t, t>0$, given some initial distribution $P(\mathbf{n}, 0)$. The probability distribution is then taken to evolve according to a master equation of the form $[29-31]$

$$
\begin{gathered}
\frac{d P(\mathbf{n}, t)}{d t}=\sum_{k=1}^{M} \sum_{r= \pm 1}\left[T_{k, r}\left(\mathbf{n}-r \mathbf{e}_{k}\right) P\left(\mathbf{n}-r \mathbf{e}_{k}, t\right)\right. \\
\left.-T_{k, r}(\mathbf{n}) P(\mathbf{n}, t)\right]
\end{gathered}
$$

Here $\mathbf{e}_{k}$ denotes the unit vector whose $k$ th component is equal to unity. The corresponding transition rates are given by

$T_{k,-1}(\mathbf{n})=\alpha_{k} n_{k}, T_{k,+1}(\mathbf{n})=N f\left(\sum_{l} w_{k l} n_{l} / N+h_{k}\right)$,

where $\alpha_{k}$ are rate constants, $w_{k l}$ is the effective synaptic weight from the $l$ th to the $k$ th population, and $h_{k}$ are external inputs. Equation (4.1) is supplemented by the boundary conditions $P(\mathbf{n}, t) \equiv 0$ if $n_{i}=-1$ for some $i$. The master equation preserves the normalization condition $\sum_{n_{1} \geq 0} \sum_{n_{M} \geq 0} P(\mathbf{n}, t)=1$ for all $t \geq 0$.
Introduce the rescaled variables $x_{k}=n_{k} / N$ and corresponding transition rates

$$
\Omega_{k,-1}(\mathbf{x})=\alpha_{k} x_{k}, \Omega_{k, 1}(\mathbf{x})=f\left(\sum_{l} w_{k l} x_{l}+h_{k}\right) .
$$

Carrying out a Kramers-Moyal expansion to second order then leads to the multivariate FP equation

$$
\begin{aligned}
\frac{\partial P(\mathbf{x}, t)}{\partial t}= & -\sum_{k=1}^{M} \frac{\partial}{\partial x_{k}}\left[A_{k}(\mathbf{x}) P(\mathbf{x}, t)\right] \\
& +\frac{\epsilon^{2}}{2} \sum_{k=1}^{M} \frac{\partial^{2}}{\partial x_{k}^{2}}\left[B_{k}(\mathbf{x}) P(\mathbf{x}, t)\right]
\end{aligned}
$$

with $\epsilon=N^{-1 / 2}$,

$$
A_{k}(\mathbf{x})=\Omega_{k, 1}(\mathbf{x})-\Omega_{k,-1}(\mathbf{x})
$$

and

$$
B_{k}(\mathbf{x})=\Omega_{k, 1}(\mathbf{x})+\Omega_{k,-1}(\mathbf{x}) .
$$

The solution to the Fokker-Planck equation (4.4) determines the probability density function for a corresponding stochastic process $\mathbf{X}(t)=\left(X_{1}(t), \ldots, X_{M}(t)\right)$, which evolves according to a neural Langevin equation of the form

$$
d X_{k}=A_{k}(\mathbf{X}) d t+\epsilon b_{k}(\mathbf{X}) d W_{k}(t)
$$

with $b_{k}(\mathbf{x})^{2}=B_{k}(\mathbf{x})$. Here $W_{k}(t)$ denotes an independent Wiener process such that

$$
\left\langle W_{k}(t)\right\rangle=0, \quad\left\langle W_{k}(t) W_{l}(s)\right\rangle=\delta_{k, l} \min (t, s) .
$$

As in the one-population model, the FP or Langevin equation captures the relatively fast stochastic dynamics within the basin of a attraction of a stable fixed point (or limit cycle) of the corresponding deterministic rate equations

$$
\frac{d u_{k}}{d t}=-\alpha_{k} u_{k}+f\left(\sum_{l} w_{k l} u_{l}+h_{k}\right) \text {. }
$$

However, in order to analyze state transitions between basins of attraction, it is necessary to consider a higherdimensional version of the asymptotic methods presented in section III. In this section we first show how the neural Langevin equation can be used to analyze noiseinduced oscillations in a two-population network. We then describe how the WKB approximation and associated Hamiltonian formulation can be extended to multiple-populations. 


\section{Noise-induced amplification of oscillations in an E-I network}

One of the major differences between the single and multi-population models is that the latter can support limit cycle oscillations. The simplest and best studied example is a two-population model consisting of an excitatory population interacting with an inhibitory population as shown in Fig. 8. The associated mean-field equation (4.9) for this E-I network reduces to the pair of equations

$$
\begin{aligned}
\frac{d u_{E}}{d t} & =-u_{E}+f\left(w_{E E} u_{E}-w_{E I} u_{I}+h_{E}\right) \\
\frac{d u_{I}}{d t} & =-u_{I}+f\left(w_{I E} u_{E}-w_{I I} u_{I}+h_{I}\right),
\end{aligned}
$$

where we have set $\alpha_{E, I}=1$ for simplicity. The bifurcation structure of the two-population Wilson-Cowan model given by equations (4.10) has been analyzed in detail elsewhere [50]. An equilibrium $\left(u_{E}^{*}, u_{I}^{*}\right)$ is obtained as a solution of the pair of equations

$$
\begin{aligned}
u_{E}^{*} & =f\left(w_{E E} u_{E}^{*}-w_{E I} u_{I}^{*}+h_{E}\right) \\
u_{I}^{*} & =f\left(w_{I E} u_{E}^{*}-w_{I I} u_{I}^{*}+h_{I}\right) .
\end{aligned}
$$

These can be inverted to yield

$$
\begin{aligned}
h_{E} & =f^{-1}\left(u_{E}^{*}\right)-w_{E E} u_{E}^{*}+w_{E I} u_{I}^{*} \\
h_{I} & =f^{-1}\left(u_{I}^{*}\right)-w_{I E} u_{E}^{*}+w_{I I} u_{I}^{*} .
\end{aligned}
$$

As a simplification, let us take the gain function $f$ to

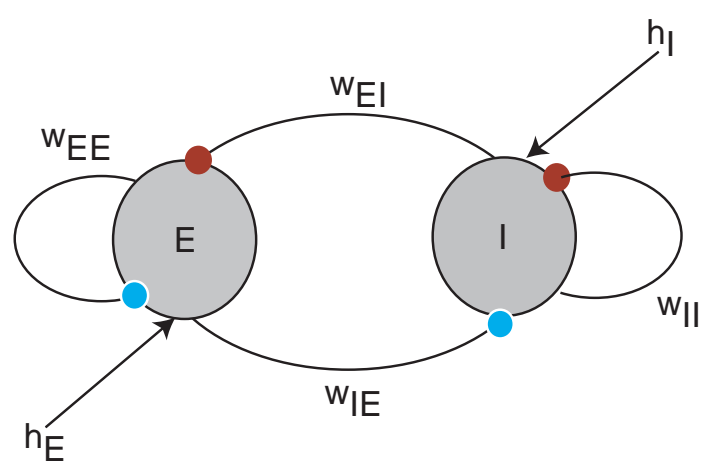

FIG. 8: Two-population E-I network

be the simple sigmid $f(u)=\left(1+\mathrm{e}^{-u}\right)^{-1}$. Using the fact that the sigmoid function then satisfies $f^{\prime}=f(1-f)$ and applying the fixed point equations allows us to represent the associated Jacobian in the form

$\mathbf{J}=\left(\begin{array}{cc}-1+w_{E E} u_{E}^{*}\left(1-u_{E}^{*}\right) & -w_{E I} u_{E}^{*}\left(1-u_{E}^{*}\right) \\ w_{I E} u_{I}^{*}\left(1-u_{I}^{*}\right) & -1-w_{I I} u_{I}^{*}\left(1-u_{I}^{*}\right)\end{array}\right)$.

An equilibrium will be stable provided that the eigenvalues $\lambda_{ \pm}$of $\mathbf{J}$ have negative real parts, where

$$
\lambda_{ \pm}=\frac{1}{2}\left(\operatorname{Tr} \mathbf{J} \pm \sqrt{[\operatorname{Tr} \mathbf{J}]^{2}-4 \operatorname{Det} \mathbf{J}}\right) .
$$

This leads to the stability conditions $\operatorname{Tr} \mathbf{J}<0$ and Det $\mathbf{J}>0$. In order to construct a phase diagram in the $\left(h_{E}, h_{I}\right)$-plane for a fixed weight matrix $\mathbf{w}$, we express $u_{I}^{*}$ as a function of $u_{E}^{*}$ by imposing a constraint on the eigenvlaues $\lambda_{ \pm}$and then substitute the resulting function into equations (4.12). This yields bifurcation curves in the $\left(h_{E}, h_{I}\right)$-plane that are parameterized by $u_{E}^{*}, 0<u_{E}^{*}<1$, see Fig. 9. For example, the constraint

$$
\operatorname{Tr} \mathbf{J} \equiv-2+w_{E E} u_{E}^{*}\left(1-u_{E}^{*}\right)-w_{I I} u_{I}^{*}\left(1-u_{I}^{*}\right)=0
$$

with Det $\mathbf{J}>0$ determines Hopf bifurcation curves where a pair of complex conjugate eigenvalues cross the imaginary axis. Since the trace is a quadratic function of $u_{E}^{*}, u_{I}^{*}$, we obtain two Hopf branches. Similarly, the constraint Det $\mathbf{J}=0$ with $\operatorname{Tr} \mathbf{J}<0$ determines saddle-node or fold bifurcation curves where a single real eigenvalue crosses zero. The saddle-node curves have to be determined numerically, since the determinant is a quartic function of $u_{E}^{*}, u_{I}^{*}$.

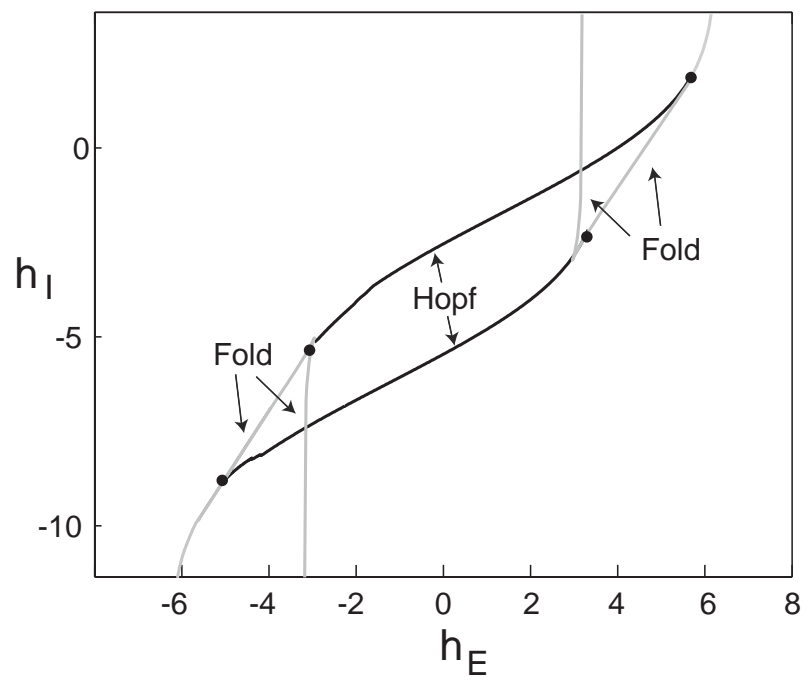

FIG. 9: Phase diagram of two-population Wilson-Cowan model (4.10) for fixed set of weights $w_{E E}=w_{I E}=w_{E I}=$ $10, w_{I I}=4$. The dots correspond to Takens-Bogdanov bifurcation points

Consider a point in parameter space where there exists a single fixed point of the mean-field equations (4.10). Suppose that the fixed point is a stable focus so that its Jacobian has a pair of complex conjugate eigenvalues. The corresponding deterministic network exhibits transient or subthreshold oscillations that decay at a rate given by the real part of the eigenvalues. We will show how intrinsic noise can convert these transient oscillations into large amplitude sustained coherent oscillations, giving rise to a peak in the corresponding power spectrum (quasi-cycles). We proceed along similar lines to recent work on biochemical oscillations in cellular systems $[40,41]$. The first step is to decompose the solution to the neural Langevin equation (4.7) as

$$
X_{k}=u_{k}^{*}+\epsilon \eta_{k}(t)
$$



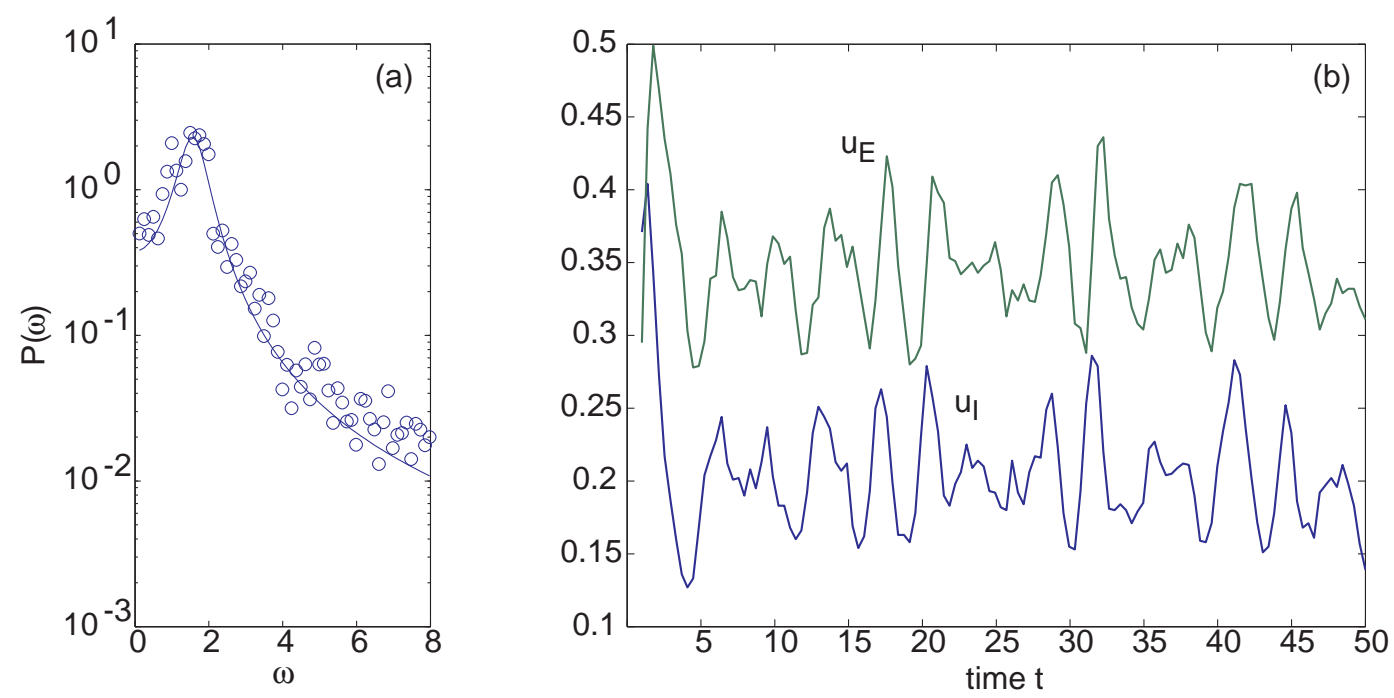

FIG. 10: Noise-amplification of subthreshold oscillations in an E-I network. (a) Power spectrum of fluctuations (solid curve) obtained by Fourier transforming the Langevin equation (4.18). Data points are from stochastic simulations of full master equation with $N=1000$, averaged over 100 samples. (b) Sample trajectories obtained from stochastic simulations of full master equation with $N=100$. Same weight parameter values as Fig. 9 with $h_{E}=0, h_{I}=-2$.

such that $A_{k}\left(\mathbf{u}^{*}\right)=0$ where $\mathbf{u}=\left(u_{E}, u_{I}\right)$. Taylor expanding equation (4.7) to first order in $\epsilon$ then implies that $\eta_{k}$ satisfies the linear Langevin equation

$$
d \eta_{k}=\sum_{l=E, I} J_{k l} \eta_{l} d t+b_{k}\left(\mathbf{u}^{*}\right) d W_{k}(t),
$$

where $J_{k l}=\partial A_{k} /\left.\partial x_{l}\right|_{\mathbf{x}=\mathbf{u}^{*}}$. Introducing the white noise processes $\xi_{k}(t)$ according to $d W_{k}(t)=\xi_{k}(t) d t$ with

$$
\left\langle\xi_{k}(t)\right\rangle=0, \quad\left\langle\xi_{k}(t) \xi_{l}\left(t^{\prime}\right)\right\rangle=\delta_{k, l} \delta\left(t-t^{\prime}\right),
$$

we can rewrite the Langevin equation in the form

$$
\frac{d \eta_{k}}{d t}=\sum_{l=E, I} J_{k l} \eta_{l}+b_{k}\left(\mathbf{u}^{*}\right) \xi_{k}(t),
$$

Let $\widetilde{\eta}_{k}(\omega)$ denote the Fourier transform of $\eta(t)$ with

$$
\widetilde{\eta}_{k}(\omega)=\int_{-\infty}^{\infty} \mathrm{e}^{-i \omega t} \eta_{k}(t) d t
$$

Taking Fourier transforms of equation (4.18) gives

$$
-i \omega \widetilde{\eta}_{k}(\omega)=\sum_{l=E, I} J_{k l} \widetilde{\eta}_{l}(\omega)+b_{k}\left(\mathbf{u}^{*}\right) \widetilde{\xi}_{k}(\omega) .
$$

This can be rearranged to give

$$
\widetilde{\eta}_{k}(\omega)=\sum_{l=E, I} \Phi_{k l}^{-1}(\omega) b_{l}\left(\mathbf{u}^{*}\right) \widetilde{\xi}_{l}(\omega)
$$

with $\Phi_{k l}(\omega)=-i \omega \delta_{k, l}-J_{k l}$. The Fourier transform of the white noise process $\xi_{k}(t)$ has the correlation function

$$
\left\langle\widetilde{\xi}_{k}(\omega) \widetilde{\xi}_{l}(\omega)\right\rangle=2 \pi \delta_{k, l} \delta\left(\omega+\omega^{\prime}\right) .
$$

The power spectrum is defined according to

$$
2 \pi \delta(0) P_{k}(\omega)=\left\langle\left|\widetilde{\eta}_{k}(\omega)\right|^{2}\right\rangle,
$$

so that equations (4.20) and (4.21) give

$$
P_{k}(\omega)=\sum_{l=E . I}\left|\Phi_{k l}^{-1}(\omega)\right|^{2} B_{l}\left(\mathbf{u}^{*}\right),
$$

with $B_{l}\left(\mathbf{u}^{*}\right)=b_{l}\left(\mathbf{u}^{*}\right)^{2}$. The right-hand side can be evaluated explicitly to yield

$$
P_{k}(\omega)=\frac{\beta_{k}+\gamma_{k} \omega^{2}}{|D(\omega)|^{2}}
$$

with $D(\omega)=\operatorname{Det} \Phi(\omega)=-\omega^{2}+i \omega \operatorname{Tr} \mathbf{J}+\operatorname{Det} \mathbf{J}$ and

$$
\begin{aligned}
\beta_{E}=J_{22}^{2} B_{E}\left(\mathbf{u}^{*}\right)+J_{12}^{2} B_{I}\left(\mathbf{u}^{*}\right), & \gamma_{E}=B_{E}\left(\mathbf{u}^{*}\right) \\
\beta_{I}=J_{21}^{2} B_{E}\left(\mathbf{u}^{*}\right)+J_{11}^{2} B_{I}\left(\mathbf{u}^{*}\right), & \gamma_{I}=B_{I}\left(\mathbf{u}^{*}\right) .
\end{aligned}
$$

Finally, evaluating the denominator and using the stability conditions $\operatorname{Tr} \mathbf{J}<0$, Det $\mathbf{J}>0$, we have [40]

$$
P_{k}(\omega)=\frac{\beta_{k}+\gamma_{k} \omega^{2}}{\left(\omega^{2}-\Omega_{0}^{2}\right)^{2}+\Gamma^{2} \omega^{2}}
$$

where we have set $\Gamma=\operatorname{Tr} \mathbf{J}$ and $\Omega_{0}^{2}=\operatorname{Det} \mathbf{J}$.

In Fig. 10, we compare our analytical calculation of the spectrum for subthreshold oscillations in an E-I pair with direct simulations of the full master equation (2.3). We choose a point in parameter space that lies just above the upper Hopf curve in Fig. 9 by taking $h_{E}=0, h_{I}=-2$. The only fixed point is a stable focus. It can be seen in Fig. 10(a) that the spectrum exhibits a strong peak at a 
frequency $\omega \approx 2$, which is close to the Hopf frequency of limit cycle oscillations generated by crossing the nearby Hopf bifurcation boundary by decreasing the inhibitory input $h_{I}$. Sample trajectories for $u_{E}$ and $u_{I}$ also indicate oscillatory-like behavior, see Fig. 9(b).

Note that it is also possible to extend the above spectral analysis to the case where the mean field equations support a limit cycle oscillation by using Floquet theory [41]. However, a more relevant approach within the neural context would be to carry out a phase reduction of the underlying Langevin equation after converting it from the Ito to Stratanovich interpretation (so the standard rules of calculus can be applied [45]). This would then provide a framework for studying the effects of intrinsic noise on the synchronization of synaptically coupled E-I networks, each of which exhibits limit cycle oscillations in the mean field limit. One application of such a model is to stimulus-induced oscillations and synchrony in primary visual cortex model, where each E-I network is interpreted as a cortical column consisting of reciprocally coupled populations of excitatory and inhibitory neurons $[46,47]$.

\section{Metastable states and the WKB approximation for multiple populations}

It is clear from Fig. 9 that there also exist parameter regimes in which the deterministic E-I network exhibits bistability due to the emergence of an additional stable fixed point via a fold or saddle-node bifurcation. Unfortunately, the analysis of metasability in a stochastic multi-population model is considerably more involved than the one-population case. Here we briefly describe how to generalize the WKB method of section III; see Refs. $[35,42,43]$ for more details. Let $\Pi(\mathbf{x}), \mathbf{x}=\mathbf{n} / N$, denote the quasistationary probability distribution associated with finding the network in the neighborhood of a stable fixed point $S$ of the mean field equations (4.9). That is,

$$
\begin{gathered}
\sum_{k=1}^{M} \sum_{r= \pm 1}\left[\Omega_{k, r}\left(\mathbf{x}-r \mathbf{e}_{k} / N\right) \Pi\left(\mathbf{x}-r \mathbf{e}_{k} / N\right)\right. \\
\left.-\Omega_{k, r}(\mathbf{x}) \Pi(\mathbf{x})\right]=0 .
\end{gathered}
$$

Following the analysis of the one-population model, we seek a solution that has the WKB form

$$
\Pi(\mathbf{x}) \sim K(\mathbf{x}) \mathrm{e}^{-N W(\mathbf{x})}, \quad K(S)=1, W(S)=0
$$

Substituting into the stationary master equation (4.28) and collecting $\mathcal{O}(1)$ terms gives the Hamilton-Jacobi equation

$$
H(\mathbf{x}, \mathbf{p}) \equiv \sum_{r= \pm 1} \sum_{k=1}^{M} \Omega_{k, r}(\mathbf{x})\left[\mathrm{e}^{r p_{k}}-1\right]=0
$$

where $p_{k}=\partial W / \partial x_{k}$. Similarly, collecting $\mathcal{O}(1 / N)$ terms generates a differential equation for the factor $K$,

$$
\sum_{i} \frac{\partial H}{\partial p_{i}} \frac{\partial K}{\partial x_{i}}=-\left[\sum_{i} \frac{\partial^{2} H}{\partial p_{i} \partial x_{i}}+\frac{1}{2} \sum_{i, j} \frac{\partial^{2} W}{\partial x_{i} x_{j}} \frac{\partial^{2} H}{\partial p_{i} \partial p_{j}}\right] K .
$$

Under the corresponding classical mechanical interpretation, the associated Hamiltonian $H$ determines the motion of a particle with position vector $\mathbf{x} \in \mathbb{R}^{M}$ and conjugate momentum vector $\mathbf{p}$. A trajectory of the particle parameterized by $t$ is given by the solution of Hamilton's equations

$$
\begin{aligned}
& \dot{x}_{k}=\frac{\partial H}{\partial p_{k}}=\sum_{r= \pm 1} r \Omega_{k, r}(\mathbf{x}) \mathrm{e}^{r p_{k}} \\
& \dot{p}_{k}=-\frac{\partial H}{\partial x_{k}}=\sum_{r= \pm 1} \sum_{l=1}^{M} \frac{\partial \Omega_{l, r}}{\partial x_{k}}(\mathbf{x})\left[\mathrm{e}^{r p_{l}}-1\right]
\end{aligned}
$$

given the initial conditions $\left(\mathbf{x}\left(t_{0}\right), \mathbf{p}\left(t_{0}\right)\right)$ in phase-space. Introducing the Lagrangian

$$
L(\mathbf{x}, \dot{\mathbf{x}})=\mathbf{p} \cdot \dot{\mathbf{x}}-H(\mathbf{x}, \mathbf{p})
$$

it follows that $W(\mathbf{x})$ with $W(S)=0$ corresponds to the classical action evaluated along the least-action trajectory from $S$ to $x[42]$ :

$$
W(\mathbf{x})=\inf _{\mathbf{x}\left(t_{0}\right)=S, \mathbf{x}(T)=\mathbf{x}} \int_{0}^{T} L(\mathbf{x}, \dot{\mathbf{x}}) d t .
$$

Since $p_{k}=\partial_{k} W$ everywhere along this path, it follows that

$$
W(\mathbf{x})=\int_{S}^{\mathbf{x}} p\left(\mathbf{x}^{\prime}\right) \cdot d \mathbf{x}^{\prime}
$$

with the line integral taken along the trajectory. Since $S$ is a fixed point of the mean-field equation (4.9), that is, $\sum_{r= \pm 1} r \Omega_{k, r}(S)=0$ for all $k=1, \ldots, M$, it can also be shown that $(S, 0)$ is a hyperbolic fixed point of the Hamiltonian dynamical system given by equations (4.32) and (4.33). It follows that along any most probable functional path starting from $S$ we have $\lim _{t \rightarrow-\infty}(\mathbf{x}(t), \mathbf{p}(t))=$ $(S, 0)$ so that one should take $t_{0}=-\infty$ in equation (4.35). From a dynamical systems perspective, the most probable paths form the unstable manifold of the fixed point $(S, 0)$. The stable manifold is given by $\mathbf{p}=0$ and consists of trajectories given by solutions of the mean-field equation (4.9).

In contrast to the one population model, it is no longer possible to obtain analytical expressions for the quasistationary density. However, one can numerically evaluate $W(\mathbf{x})$ and $K(\mathbf{x})$ by evolving Hamilton's equations from initial points $(\mathbf{x}, \mathbf{p}(\mathbf{x}))$ in a neighborhood of $(S, 0)$ with $\mathbf{p}(\mathbf{x})$ determined using a Gaussian approximation of $W$. Let $Z_{i j}=\partial_{i} \partial_{j} W(\mathbf{x})$ denote the Hessian matrix of $W(\mathbf{x})$ 
[38]. A dynamical equation for $\mathbf{Z}$ can be derived by differentiating equation $H(\mathbf{x}, \mathbf{p}(\mathbf{x}))=0$ twice with respect to $\mathbf{x}$ using Hamilton's equations and

$$
\frac{d}{d x_{k}}=\frac{\partial}{\partial x_{k}}+\sum_{l} \frac{d p_{l}}{d x_{k}} \frac{\partial}{\partial p_{l}} .
$$

This yields the matrix equation

$$
\dot{Z}_{i j} \equiv \sum_{k} \frac{\partial H}{\partial p_{k}} \frac{\partial Z_{i j}}{\partial x_{k}}=-\left[\sum_{k, l} \frac{\partial^{2} H}{\partial p_{k} \partial p_{l}} Z_{i k} Z_{j l}+\sum_{l} \frac{\partial^{2} H}{\partial x_{i} \partial p_{l}} Z_{j l}+\sum_{l} \frac{\partial^{2} H}{\partial x_{j} \partial p_{l}} Z_{i l}+\frac{\partial^{2} H}{\partial x_{i} \partial x_{j}}\right]
$$

At the fixed point $(S, 0)$ we have $\dot{\mathbf{Z}}=0$ and

$$
\frac{\partial^{2} H}{\partial x_{l} \partial p_{k}}=\sum_{r= \pm 1} r \frac{\partial \Omega_{k, r}}{\partial x_{l}}(S) \equiv A_{k l}, \quad \frac{\partial^{2} H}{\partial p_{k} \partial p_{l}}=\delta_{k, l} \sum_{r= \pm 1} \Omega_{k, r}(S) \equiv B_{k l}, \quad \frac{\partial^{2} H}{\partial x_{k} \partial x_{l}}=0
$$

so that the Hessian evaluated at $S$ is given by the solution to the algebraic Riccati equation

$$
\mathbf{Z}(S) \mathbf{B Z}(S)+\mathbf{Z}(S) \mathbf{A}+\mathbf{A}^{t} \mathbf{Z}(S)=0 .
$$

It can be shown that if $\operatorname{Tr} \mathbf{A} \neq 0$ then there are exactly four solutions for $\mathbf{Z}(S)$ : one of full rank, two of unit rank, and the trivial solution $\mathbf{Z}(S)=0$ [43]. Since the point $S$ is a local minimum of $W(\mathbf{x})$, it follows that only the full rank solution is physically relevant and that in a small neighborhood of $S$ we have the Gaussian approximation

$$
W(\mathbf{x}) \approx-\frac{1}{2} \sum_{i, j} Z_{i j}(S)\left(x_{i}-x_{i}(S)\right)\left(x_{j}-x_{j}(S)\right)
$$

with $\mathbf{Z}(S)$ the unique solution to the Riccati equation. It follows that $p_{i}(\mathbf{x}) \approx \sum_{j} Z_{i j}(S)\left(x_{j}-x_{j}(S)\right)$ and

$\Pi(\mathbf{x}) \sim \exp \left(-\frac{1}{2} \sum_{i, j} Z_{i j}(S)\left(x_{i}-x_{i}(S)\right)\left(x_{j}-x_{j}(S)\right)\right)$.

The latter is also the solution to the corresponding Fokker-Planck equation (4.4), linearized about the fixed point $S[3]$.

Having obtained the WKB approximation that matches the Gaussian approximation around $S$, it remains to match with an inner solution close to the boundary $\partial \mathcal{M}$ of the basin of attraction. This is necessary, since the WKB solution does not satisfy the appropriate boundary condition on $\partial \mathcal{M}$. Typically, one either takes an absorbing boundary condition $P_{0}(\mathbf{x})=0$ on $\partial \mathcal{M}$ or one assumes that there is a constant flux $J(\mathbf{x})$ through the boundary that is determined self-consistently. Following standard problems in classical transition rate theory, suppose that the deterministic network described by the mean-field equation (4.9) is bistable so that there exist two stable fixed points $S, S^{\prime}$ separated by a saddle point $Q$ that lies on the separatrix $\partial \mathcal{M}$. In this case the most probable path from $S$ to $S^{\prime}$ is through $Q$ and the behavior around $Q$ dominates the matched asymptotics. Provided that certain additional constraints hold, it can be shown that the transition rate takes the form [43]

$$
\lambda_{0}=\frac{\lambda_{+}(Q)}{2 \pi}\left[\frac{\operatorname{det} \mathbf{Z}(S)}{\operatorname{det} \mathbf{Z}(Q)}\right]^{1 / 2} K(Q) \mathrm{e}^{-W(Q) / N},
$$

where $\lambda_{+}(Q)$ is the positive eigenvalue of the Jacobian obtained by the linearizing the mean-field equation about $Q$. In general, the geometry of the trajectories in phase space make the calculation of transition rates in the multi-dimensional case computationally challenging. However, an efficient numerical method called "transition path sampling" has recently been developed that allows one to study transition pathways for rare events in complex systems which can then be used to extract important physical quantities such as transition rates from metastable states [51].

\section{Continuum neural field model}

One of the distinct features of neural systems compared to chemical systems is that when spatial degrees of freedom are taken into account, the former involves nonlocal interactions rather than diffusive interactions. That is, it is common to model large-scale cortical networks in terms of spatially distributed populations labelled by spatial coordinates $\mathbf{q} \in \mathbb{R}^{2}$. In the deterministic limit, these continuum networks evolve according to neural field equations of the form [34] 


$$
\frac{\partial u_{k}(\mathbf{q}, t)}{\partial t}=-\alpha_{k} u_{k}(\mathbf{q}, t)+f\left(\sum_{l=E, I} \int w_{k l}\left(\mathbf{q}, \mathbf{q}^{\prime}\right) u_{l}\left(\mathbf{q}^{\prime}, t\right) d \mathbf{q}^{\prime}+h_{k}(\mathbf{q}, t)\right)
$$

where $k, l=E, I$ specifies whether a given population is excitatory or inhibitory. The synaptic weight distributions $w_{k l}\left(\mathbf{q}, \mathbf{q}^{\prime}\right)$ specify the form of the nonlocal interactions. The associated stochastic neural field model is described in terms of a probability functional satisfying an infinite-dimensional version of the master equation (4.1). Under the diffusion approximation, this reduces to a functional $\mathrm{FP}$ equation of the form

$$
\begin{aligned}
\frac{\partial P([\mathbf{x}], t)}{\partial t}= & -\sum_{k=E, I} \int \frac{\delta}{\delta x_{k}(\mathbf{q})}\left[A_{k, \mathbf{q}}([\mathbf{x}]) P([\mathbf{x}], t)\right] \\
& +\frac{\epsilon^{2}}{2} \sum_{k=E, I} \frac{\delta^{2}}{\delta x_{k}(\mathbf{q})^{2}}\left[B_{k, \mathbf{q}}([\mathbf{x}]) P([\mathbf{x}], t)\right],
\end{aligned}
$$

with $[\mathbf{x}]=\left\{\left(x_{E}(\mathbf{q}), x_{I}(\mathbf{q})\right), \mathbf{q} \in \mathbb{R}^{2}\right\}$,

$$
\begin{aligned}
& A_{k, \mathbf{q}}([\mathbf{x}])=-\alpha_{k} x_{k}(\mathbf{q})+f_{k, \mathbf{q}}([\mathbf{x}]), \\
& B_{k, \mathbf{q}}([\mathbf{x}])=\alpha_{k} x_{k}(\mathbf{q})+f_{k, \mathbf{q}}([\mathbf{x}]),
\end{aligned}
$$

and

$f_{k, \mathbf{q}}([\mathbf{x}])=f\left(\sum_{l=E, I} \int w_{k l}\left(\mathbf{q}, \mathbf{q}^{\prime}\right) x_{l}\left(\mathbf{q}^{\prime}, t\right) d \mathbf{q}^{\prime}+h_{k}(\mathbf{q}, t)\right)$.

It is has previously been shown that deterministic neural field models support a variety of spatially coherent states including spatially periodic patterns arising via Turing-like instabilities [34, 52-55]. In the case of homogeneous weight distributions, one could apply Fourier methods to the FP equation (4.44) in order to analyze the effects of intrinsic noise on such pattern forming instabilities [48]. One could also extend the analysis of quasicycles to the spatial domain, following along similar lines to a recent study of a diffusive predator-prey model [56]. However, as in the case of finite population models, the FP equation (4.44) would not take proper account of noise-induced transitions to other metastable states.

\section{DISCUSSION}

In this paper we have shown how techniques from the study of chemical master equations can be carried over to that of a scaled version of the neural master equation introduced by Buice et. al. [30, 31]. The latter can be viewed as a stochastic version of the Wilson-Cowan model of neural population dynamics in which noise is treated intrinsically. We focused on two particular effects of intrinsic noise fluctuations. First, we calculated exponentially small escape rates between metastable states of a one-population model using a WKB approximation and matched asymptotics; corrections to mean-field equations based on a system-size expansion (or loop expansion in the corresponding path integral formulation) are no longer appropriate. Second, we analyzed quasicycles in an E-I network by determining the power spectrum of the associated neural Langevin equation.

A major outstanding question is to what extent the master equation formulation captures the types of statistical fluctuations in population activity that can be observed in real nervous tissue. Buice and Cowan [30] have used path integral methods and renormalization group theory to establish that a continuum version of the stochastic Wilson-Cowan model belongs to the universality class of directed percolation, and consequently exhibits power law behavior suggestive of many measurements of spontaneous cortical activity in vitro and in vivo $[57,58]$. However, the existence of power law behavior is controversial [59]. Moreover, it is likely that a variety of different stochastic models could exhibit behavior consistent with what is observed experimentally. Therefore, can one use multi-scale analysis to derive a master equation of population dynamics (or perhaps a different type of stochastic Wilson-Cowan model) starting from more detailed microscopic models? That is, the master equation formulation of stochastic neurodynamics developed here and elsewhere [29-31] is a phenomenological representation of stochasticity at the population level. It is not derived from a detailed microscopic model of synaptically coupled spiking neurons, and it is not yet clear under what circumstances such a microscopic model would yield population activity consistent with the master equation approach. Nevertheless, if one views the Wilson-Cowan rate equations $[33,34]$ as an appropriate description of large-scale neural activity in the deterministic limit, it is reasonable to explore ways of adding noise to such equations from a top-down perspective. One possibility is to consider a Langevin version of the WilsonCowan equations involving some form of extrinsic spatiotemporal white noise $[48,49]$, whereas another is to view the Wilson-Cowan rate equations as the thermodynamic limit of an underlying master equation that describes the effects of intrinsic noise [29-32]. As we have highlighted in this paper, the two formulations are not equivalent.

One possible application of the master equation formulation of neural population dynamics is modeling noiseinduced switching during binocular rivalry. Binocular 
rivalry concerns the phenomenon whereby perception switches back and forth between different images presented to either eye. Recordings from the brain and reports by human subjects during binocular rivalry tasks show eye dominance time statistics that may be fit to a gamma distribution [62]. In addition, statistical analysis of such data shows little correlation between one dominance time and the next [62-64]. This suggests that the switching between one eye's dominance and the next may be largely driven by a stochastic process. One possibility is that the inputs arriving at the network encoding rivalry are stochastic, so that the noise is extrinsic. A number of recent models have examined dominance switching times due to additive noise terms in a competitive Wilson-Cowan model with additional slow adapting variables [65-67]. On the other hand, Laing and Chow [68] considered a deterministic spiking neuron model of binocular rivalry in which the statistics of the resulting dominance times appeared noisy due to the aperiodicity of the high-dimensional system's trajectories. The latter is suggestive of an effective intrinsic noise source within a rate-based population model. One important issue is whether the switching time statistics is better captured by noise-induced transitions in a bistable network or noise-induced amplification of subthreshold oscillations (quasicycles). In order to study this within the master equation framework, it will be necessary to incorporate some form of slow adaptation into the stochastic Wilson-Cowan model.

\section{Acknowledgements}

This publication was based on work supported in part by the National Science Foundation (DMS-0813677) and by Award No KUK-C1-013-4 made by King Abdullah University of Science and Technology (KAUST). PCB was also partially supported by the Royal Society Wolfson Foundation.
[1] P. S. Swain, M. B. Elowitz and E. D. Siggia. Proc. Natl. Acad. Sci. USA 9912795 (2002).

[2] J. Paulsson. Nature 427415 (2004).

[3] N. G. Van Kampen. Stochastic processes in physics and chemistry. North-Holland, Amsterdam (1992).

[4] T. G. Kurtz. Math.Prog. Stud. 567 (1976).

[5] P. Haangi, H. Grabert, P. Talkner and H. Thomas. Z. Physik B 28135 (1984).

[6] F. Baras, M. M. Mansour and J. E. Pearson. J. Chem. Phys. 1058257 (1996).

[7] M. Vellela and H. Qian. J. R. Soc. Interface 6925 (2010).

[8] P. S. Swain and A. Longtin. Chaos 16026101 (2006).

[9] A. A. Faisal, L. P. J. Selen and D. M. Wolpert. Nat. Rev. Neurosci., 9292 (2008).

[10] W. R. Softky and C. Koch. J. Neurosci., 13334 (1993).

[11] K. Pakdaman, M. Thieullen and G. Wainrib. J. Appl. Prob. In press 2010.

[12] D. Cai, L. Tao, M. Shelley, and D. W. McLaughlin. Proc. Natl. Acad. Sci. USA, 1017757 (2004).

[13] A. V. Rangan, G. Kovacic, and D. Cai. Phys. Rev. E 77 041915 (2008).

[14] D. Nykamp and D. Tranchina. J. Comp. Neurosci., 819 (2000).

[15] A. Omurtag, B. W. Knight, and L. Sirovich. J. Comput. Neurosci. 851 (2000).

[16] C. Ly and D. Tranchina. Neural Comput., 192032 (2007).

[17] L. F. Abbott and C. van Vresswijk. Phys. Rev. E 48 1483 (1993).

[18] W. Gerstner and J. L. Van Hemmen. Phys. Rev. Lett., 71312 (1993).

[19] N. Brunel and V. Hakim. Neural Comput., 111621 (1999).

[20] N. Brunel. J Comput. Neurosci 8183 (2000).

[21] A. Renart, N. Brunel, and X.-J. Wang. In J. Feng, editor, Computational Neuroscience: a comprehensive approach,pp. 431-490. CRC Press, Boca Raton, Fl (2004).
[22] C. Meyer and C. van Vreeswijk. Neural Comput. 14369 (2002).

[23] M. Mattia and P. Del Guidice. Phys. Rev. E, 66051917 (2002).

[24] H. Soula and C. C. Chow. Neural Comput, 193262 (2007).

[25] S. El Boustani and A. Destexhe. Neural Comput, 2146 (2009).

[26] B. Doiron, B. Lindner B, A. Longtin, L. Maler and J. Bastian. Oscillatory activity in electrosensory neurons increases with the spatial correlation of the stochastic input stimulus. Phys. Rev. Lett. 93048101 (2004).

[27] M. J. Chacron, A. Longtin and L. Maler. Delayed excitatory and inhibitory feedback shape neural information transmission. Phys. Rev. E 72051917 (2005).

[28] B. Lindner, B. Doiron and A. Longtin Theory of oscillatory firing induced by spatially correlated noise and delayed inhibitory feedback. Phys. Rev. E 72061919 (2005).

[29] P. C. Bressloff. SIAM J. Appl. Math, 701488 (2009).

[30] M. Buice and J. D. Cowan. Phys. Rev. E, 75051919 (2007).

[31] M. Buice, J. D. Cowan, and C. C. Chow. Neural Comput., 22377 (2010).

[32] T. Ohira and J. D. Cowan. In S Ellacott and I J Anderson, editors, Proceedings of the first international conference on Mathematics of neural networks, pp. 290-294. Academic Press (1997).

[33] H. R. Wilson and J. D. Cowan. Biophys. J., 121 (1972).

[34] H. R. Wilson and J. D. Cowan. Kybernetik, 1355 (1973).

[35] M. I. Dykman, E. Mori, J. Ross, and P. M. Hunt. J. Chem. Phys. A, 1005735 (1994).

[36] V. Elgart and A. Kamenev. Phys. Rev. E, 70041106 (2004).

[37] R. Hinch and S. J. Chapman. Eur. J. Appl. Math. 16427 (2005).

[38] D. M. Roma, R. A. O'Flanagan, A. E. Ruckenstein, 
A. M. Sengupta and R. Mukhopadhyay Phys. Rev. E, 71011902 (2005).

[39] C. Escudero and A. Kamanev. Phys. Rev. E, 79041149 (2009).

[40] A. J. McKane, J. D. Nagy, T. J. Newman and M. O. Stefanini J. Stat. Phys. 71165 (2007).

[41] R. P. Boland, T. Galla and A. J. McKane J. Stat. Mech., 9 P09001 (2008).

[42] M. I. Freidlin and A. D. Wentzell. Random perturbations of dynamical systems. Springer, New York (1984).

[43] R. S. Stein and D. L. Stein. SIAM J. Appl. Math 57752 (1997).

[44] C. W. Gardiner. Stochastic methods: a handbook for the natural and social sciences. 4th ed. Springer New York (2009).

[45] J. Teramae, H. Nakao and G. B. Ermentrout. Phys. Rev. Lett. 102194102 (2009).

[46] H. G. Schuster and P. Wagner. Biol. Cybern. 6477 (1990).

[47] E. R. Grannan, D. Kleinfeld, and H. Sompolinsky. Neural Comput. 5550 (1993).

[48] A. Hutt, A. Longtin and L. Schimansky-Geier, Physica D 237755 (2008).

[49] O. Faugeras, J. Touboul and B. Cessac. Fronteirs in Comp. Neurosci. 31 (2009).

[50] R. Borisyuk and A. B. Kirillov. Biol. Cybern, 66319 (1992).

[51] P. G. Bolhuis, D. Chandler, C. Dellago and P. L. Geissler. Ann. Rev. Phys. Chem. 53291 (2002).

[52] G. B. Ermentrout and J. D. Cowan, Biol. Cyber. 34137
(1979).

[53] G. B. Ermentrout. Rep. Prog. Phys. 61353 (1998).

[54] P. C. Bressloff, J. D. Cowan, M. Golubitsky, P. J. Thomas and M. Wiener, Phil. Trans. Roy. Soc. Lond. B 356299 (2001).

[55] S. Coombes, Biol. Cybern., 9391 (2005).

[56] C. A. Lugo and A. J. McKane. Phys. Rev. E 78, 051911 (2008).

[57] J. M. Beggs and D. Plenz. J. Neurosci., 245216 (2004).

[58] D. Plenz and T. C. Thiagarajan. Trends Neurosci., 30 101 (2007).

[59] C. Bedard and A. Destexhe. Biophys. J. 962589 (2009).

[60] R. Blake, A primer on binocular rivalry, including current controversies, Brain and Mind, 2 (2001), pp. 5-38.

[61] R. Blake and N. Logothetis. Nature Reviews Neurosci. 3 1 (2002).

[62] N. K. Logothetis, D. A. Leopold, and D. L. Sheinberg. Nature 380621 (1996).

[63] S. R. Lehky. Perception 17215 (1988).

[64] S. R. Lehky. Proc Roy Soc Lon. B 25971 (1995).

[65] R. Moreno-Bote, J. Rinzel, and N. Rubin. J. Neurophysiol. 981125 (2007)

[66] A. Shpiro, R. Curtu, J. Rinzel, and N. Rubin. J Neurophysiol. 97462 (2007).

[67] A. Shpiro, R. Moreno-Bote, N. Rubin, and J. Rinzel. J. Comp. Neurosci 2737 (2009).

[68] C. R. Laing and C. C. Chow. J. Comput. Neurosci. 12 39 (2002). 



\section{RECENT REPORTS}

38/10 Preconditioners for state constrained optimal control problems with Moreau-Yosida penalty function

Stoll

Wathen

39/10 Local synaptic signaling enhances the stochastic transport of

Newby motor-driven cargo in neurons

Bressloff

40/10 Convection and Heat Transfer in Layered Sloping Warm-Water Aquifer

McKibbin

Hale

Style

Walters

41/10 Optimal Error Estimates of a Mixed Finite Element Method for

Goswami

Parabolic Integro-Differential Equations with Non Smooth Initial Data

Pani

Yadav

42/10 On the Linear Stability of the Fifth-Order WENO Discretization

Motamed

Macdonald

Ruuth

43/10 Four Bugs on a Rectangle

Chapman

Lottes

Trefethen

44/10 Mud peeling and horizontal crack formation in drying clay

Style

Peppin

Cocks

45/10 Binocular Rivalry in a Competitive Neural Network with Synaptic

Kilpatrick Depression

Bressloff

46/10 A theory for the alignment of cortical feature maps during development

Bressloff

Oster

47/10 All-at-Once Solution if Time-Dependent PDE-Constrained Optimisation Problmes

Stoll

Wathen

48/10 Possible role of differential growth in airway wall remodeling in

Moulton asthma

Goriely

49/10 Variational Data Assimilation Using Targetted Random Walks

Cotter

Dashti

Robinson

Stuart

50/10 A model for the anisotropic response of fibrous soft tissues using six discrete fibre bundles

Flynn

Rubin

Nielsen

51/10 STOCHSIMGPU Parallel stochastic simulation for the Systems

Klingbeil Biology Toolbox 2 for MATLAB

Erban

Giles

Maini

52/10 Order parameters in the Landau-de Gennes theory - the static Majumdar and dynamic scenarios 
53/10 Liquid Crystal Theory and Modelling Discussion Meeting

Majumdar

Mottram

54/10 Modeling the growth of multicellular cancer spheroids in a bioengi-

Loessner neered 3D microenvironment and their treatment with an anticancer drug

Flegg

Byrne

Hall

Moroney

Clements

Hutmacher

McElwain

55/10 Scalar Z, ZK, KZK, and KP equations for shear waves in incompressible solids

Destrade

Goriely

Saccomandi

56/10 The Influence of Bioreactor Geometry and the Mechanical Environment on Engineered Tissues

Osborne

ODea

Whiteley

Byrne

Waters

57/10 A numerical guide to the solution of the bidomain equations of cardiac electrophysiology

Pathmanathan

Bernabeu

Bordas

Cooper

Garny

Pitt-Francis

Whiteley

Gavaghan

58/10 Particle-scale structure in frozen colloidal suspensions from small angle X-ray scattering

Spannuth

Mochrie

Peppin

Wettlaufer

$59 / 10 \quad$ Spin coating of an evaporating polymer solution

Munch

Please

Wagner

60/10 Stochastic synchronization of neuronal populations with intrinsic

Bressloff and extrinsic noise

Lai

Copies of these, and any other OCCAM reports can be obtained from:

Oxford Centre for Collaborative Applied Mathematics Mathematical Institute

24 - 29 St Giles' 
Oxford

OX1 3LB

England

www.maths.ox.ac.uk/occam 